\title{
MSDC-0602K, A Novel Insulin-Sensitizer Improves Insulinemia and Fatty Liver Disease Alone and in Addition to Liraglutide in Mice
}

\author{
Dakota R. Kamm ${ }^{1}$, Kelly D. Pyles ${ }^{1}$, Martin C. Sharpe ${ }^{1}$, \\ Laura Healy ${ }^{2}$, Jerry R. Colca ${ }^{3}$, Kyle S. McCommis ${ }^{1}$ \\ ${ }^{1}$ Biochemistry \& Molecular Biology, Saint Louis University School of Medicine, St. Louis, MO \\ ${ }^{2}$ LNH ToxPath Consulting LLC, Newbury Park, CA \\ ${ }^{3}$ Cirius Therapeutics, Kalamazoo, MI and San Diego, CA
}

ORCID IDs:

Dakota Kamm (0000-0003-3096-8429)

Kelly Pyles (0000-0001-9478-3365)

Martin Sharpe (0000-0002-8740-7270)

Laura Healy (0000-0001-5821-1853)

Jerry Colca (0000-0003-3933-8626)

Kyle McCommis (0000-0003-2381-1589)

Address for Correspondence: Dr. Kyle S. McCommis

431 Doisy Research Center

1100 S. Grand. Blvd.

Saint Louis, MO 63127 (USA)

Phone: 314-977-9233. Fax: 314-977-9206

kyle.mccommis@health.slu.edu

Word count: 3,972

(main text only; excludes title page, abstract, research in context, refs, funding statement, authors relationships and activities, contribution statement, and fig legends) 


\begin{abstract}
Aims/hypothesis: Insulin sensitizers and incretin mimetics are antidiabetic agents with vastly different mechanisms of action. Additionally, while thiazolidinedione (TZD) insulin sensitizers are associated with the side-effect of weight gain, glucagon-like peptide-1 receptor agonists (GLP-1RAs) can induce weight loss. We hypothesized that combination therapy with a novel TZD insulin sensitizer and the GLP-1RA Liraglutide would more significantly improve mouse models of diabetes and nonalcoholic steatohepatitis (NASH) compared to individual therapy.
\end{abstract}

Methods: $d b / d b$ mice were treated with the novel TZD MSDC-0602K by oral gavage, Liraglutide (Lira) by s.c. injection, combination $0602 \mathrm{~K}+\mathrm{Lira}$, or both vehicle solutions. Vehicle-treated $d b /+$ mice were included as non-obese controls. To assess treatment effects on nonalcoholic fatty liver disease, MSNASH mice were similarly treated with vehicle, either drug individually, or in combination. Lastly, islets were isolated from C57BL/6J mice to assess glucose-stimulated insulin secretion (GSIS).

Results: 0602K-treated $d b / d b$ mice displayed slight weight gain but completely corrected glycemia and markedly improved glucose tolerance. Lira slightly reduced body weights and modestly improved glycemia. 0602K+Lira combination still induced slight weight gain but completely corrected glycemia and improved glucose tolerance beyond lean $d b /+$ levels. As expected, 0602K resulted in reduced plasma insulin, whereas Lira further increased the hyperinsulinemia of $d b / d b$ mice. Surprisingly, 0602K+Lira treatment reduced plasma insulin and C-peptide to the same extent as mice treated with 0602K alone. 0602K did not directly reduce GSIS in isolated islets, thus the reduced insulinemia with 0602K is likely compensatory due to improved insulin action. In the MS-NASH mouse model, both 0602K or Lira alone improved plasma ALT and AST, and liver histology, but more significant improvements were observed with $0602 \mathrm{~K}+$ Lira combination therapy. 0602K or 0602K+Lira also increased pancreatic insulin content in both $d b / d b$ and MS-NASH mice.

Conclusions: MSDC-0602K corrected glycemia and reduced insulinemia when given alone, or in combination with Lira. However, $0602 \mathrm{~K}+$ Lira combination more significantly improved glucose tolerance in $d b / d b$ mice, and more significantly improved liver histology in MS-NASH mice. 


\section{Introduction}

Insulin resistance can result in elevated rates of insulin secretion and hyperinsulinemia. If unresolved, pancreatic beta cell failure can lead to loss of beta cell mass via cell death or dedifferentiation $[1,2]$. Insulin sensitizers are an attractive therapeutic strategy as they not only target the core defective insulin signaling pathways but can also reduce this stress on beta cells and preserve beta cell mass [3]. However, clinical use of the main class of insulin sensitizers, the thiazolidinediones (TZDs), is limited due to side effects such as weight gain, edema, bone loss, and bladder cancer risk. These side effects are thought to be due to agonism of the nuclear receptor peroxisome proliferatoractivated receptor $\gamma(\operatorname{PPAR} \gamma)$. Yet numerous studies have described acute, PPAR $\gamma$-independent effects of TZDs [4-7], which have led to the development of several TZDs with very low affinity for PPAR $\gamma$, such as MSDC-0602K and MSDC-0160. The molecular target of these compounds is the mitochondrial pyruvate carrier (MPC) [8-10], which is also inhibited by the traditional PPAR $\gamma$-activating TZDs [9]. The clinical profile of PPAR $\gamma$-sparing TZDs appears improved compared to traditional TZDs with respect to edema, bone loss, and degree of weight gain [11-13].

Another popular class of antidiabetic agents is the glucagon-like peptide-1 receptor agonists (GLP-1RA). These incretin-like peptides improve glycemia by increasing postprandial insulin secretion, suppressing glucagon secretion, and delaying gastric emptying. GLP-1RAs such as Liraglutide can also induce weight loss in humans and animal models $[14,15]$.

Insulin resistance and diabetes are driving factors for the development and progression of nonalcoholic fatty liver disease (NAFLD) and nonalcoholic steatohepatitis (NASH). While there are currently no approved therapies for treatment of NAFLD/NASH, both TZDs [13, 16-21] and GLP-1RAs [22-25] can improve aspects of liver pathology in humans and animal models. The purpose of this current study was to investigate whether combining the PPAR $\gamma$-sparing TZD MSDC-0602K and the GLP-1RA Liraglutide would better improve mouse models of diabetes and NASH. 


\section{$\underline{\text { Methods }}$}

All experiments and procedures were approved by the Institutional Animal Care and Use Committee of Saint Louis University, and conform to NIH guidelines for the care and use of laboratory animals [26]. All mice were housed in standard rodent caging at $20-25^{\circ} \mathrm{C}$ with ad libitum access to food and water with a light cycle from 6:00AM - 6:00PM.

\section{$d b / d b$ mouse studies}

Five-week-old male $d b / d b$ mice on C57BL/6J background, and age/sex matched $d b /+$ control mice were purchased from The Jackson Laboratory (B6.BKS(D)-Lepr ${ }^{\mathrm{db}} / \mathrm{J}$, stock \#000697; Bar Harbor, ME, USA). Mice were provided ad libitum access to water and chow (Laboratory Rodent Diet 20 (5L0B), LabDiet, St. Louis, MO, USA). At 9 weeks of age, blood glucose was measured by nick of the tail vein and measuring with a Contour Next glucometer (Ascensia Diabetes Care, Parsippany, NJ, USA). $d b / d b$ mice were then divided into treatment groups based on averaged body weights and blood glucose concentrations: Vehicle (Veh), 30mg/kg MSDC-0602K (0602K) gavage daily, 0.2mg/kg Liraglutide (Lira) s.c. injection every-other-day, or combined 0602K+Lira. Lira was obtained from MedChemExpress (HY-P0014; Monmouth Junction, NJ, USA). Vehicle gavage solution was 1\% lowviscosity carboxymethylcelluse, $0.1 \%$ Tween-80, and $1 \%$ DMSO. $0.9 \% \mathrm{NaCl}$ solution was vehicle for s.c. injection. $d b /+$ and $d b / d b$ Veh mice received both the gavage and s.c. injection vehicles. $0602 \mathrm{~K}-$ treated mice also received vehicle s.c. injection while Lira-treated mice also received gavage vehicle. After 3 weeks of treatment, mice were euthanized by $\mathrm{CO}_{2}$ asphyxiation and blood collected by cannulation of the inferior vena cava, placed into an EDTA-containing tube, and centrifuged at 8,000 x $\mathrm{g}$ for 8 minutes at $4^{\circ} \mathrm{C}$ to collect plasma. Tissues were dissected, blotted dry, frozen in liquid nitrogen, and stored at $-80^{\circ} \mathrm{C}$. Pieces of liver and pancreas were placed in neutral buffered formalin for histological evaluation.

\section{Glucose tolerance test}


After 1.5 weeks of treatment, mice were challenged by a GTT. At 8:00AM, mice were weighed and fasted. 4 hours later, mice were injected i.p. with $1 \mathrm{~g} / \mathrm{kg}$ D-glucose in $0.9 \% \mathrm{NaCl}$ immediately after measuring blood glucose $(\mathrm{T}=0)$ by a nick to the tail vein and a Contour Next glucometer (Ascensia Diabetes Care, Parsippany, NJ, USA). Blood glucose was monitored at 15, 30, 60, 90, and 120 minutes post-injection by removing the scab from the tail.

\section{MS-NASH mouse studies}

Studies of the MS-NASH mouse model (Jackson Laboratory MSNASH/PcoJ, stock \#030888) were performed at CrownBiosciences Inc. Beginning at 9 weeks of age, male MS-NASH mice were fed ad libitum with a "western diet” (D12079B, Research Diets Inc., New Brunswick, NJ, USA) containing $40 \% \mathrm{kcal}$ fat, $17 \% \mathrm{kcal}$ protein, $43 \% \mathrm{kcal}$ carbohydrate, and $1.5 \mathrm{~g} / \mathrm{kg}$ cholesterol, and ad libitum drinking water containing $5 \%(\mathrm{w} / \mathrm{v})$ fructose. Body weight was measured weekly and blood was collected via the tail vein for measurement of blood glucose and plasma alanine aminotransferase (ALT) and aspartate aminotransferase (AST) every 4 weeks as described below. Based on average ALT concentrations, mice were divided into 4 treatments: Veh, 0602K, Lira, or 0602K+Lira, performed identically to the $d b / d b$ studies described above for a duration of 6 weeks. Mice were euthanized by $\mathrm{CO}_{2}$ asphyxiation and blood collected via cardiac puncture. Tissues were dissected and frozen in liquid nitrogen. Pieces of liver and pancreas were fixed in neutral buffered formalin for histological examination.

\section{RIPCreMPC2-/- mouse studies}

Generation of $M p c 2$ floxed mice and RIPCreMPC2-/- pancreatic beta cell MPC2-/- mice on the C57BL/6J background were reported previously [10, 27]. For these studies, 6-week-old male RIPCreMPC2-/- and littermate fl/fl control mice were fed 60\% high-fat diet for 10 weeks (D12492, Research Diets Inc., New Brunswick, NJ, USA). Based on body weight, mice were divided into two single gavage treatments: Vehicle, or $30 \mathrm{mg} / \mathrm{kg} 0602 \mathrm{~K}$. 16h post gavage, mice were euthanized by $\mathrm{CO}_{2}$ asphyxiation. Blood was collected from the inferior vena cava into an EDTA-coated tube, processed as above, and plasma stored at $-80^{\circ} \mathrm{C}$. 


\section{Pancreatic islet isolation and glucose-stimulated insulin secretion assay}

Wildtype male and female C57BL/6J mice were obtained (Jackson Laboratory, stock \#000664) and maintained on normal chow. Between 10-20 weeks of age, mice were euthanized by 3-5\% isoflurane inhalation and cervical dislocation. Islets were isolated similar to previously described procedures [27, 28], after perfusion of the pancreas through the common bile duct with $5-10 \mathrm{~mL}$ of calcium-free Hanks-buffered saline solution (HBSS) supplemented with $0.4 \mathrm{mg} / \mathrm{mL}$ Type V collagenase (C9263, Sigma, St. Louis, MO, USA). After overnight culture, islets were treated with either 1 or $23 \mathrm{mM}$ glucose and either DMSO vehicle or $10 \mu \mathrm{M} 0602 \mathrm{~K}$ in a $37^{\circ} \mathrm{C}$ incubator for $1 \mathrm{~h}$. Insulin concentration of the supernatants was measured with a mouse/rat insulin ELISA (EZRMI-13K, EMD Millipore, Billerica, MA, USA).

\section{Plasma hormone, metabolite, and ALT and AST assays}

Plasma fructosamine levels were measured with a colorimetric kit (K450-100, BioVision Inc., Milpitas, CA, USA). Plasma insulin and C-peptide were measured with mouse/rat ELISAs (EZRMI13K and EZRMCP2-21K, respectively, EMD Millipore, Billerica, MA, USA). Plasma non-esterified fatty acids (NEFA) were measured by enzymatic colorimetric assay (NEFA-HR(2), FUJIFILM Wako, Mountain View, CA, USA). Plasma triacylglycerol (TAG) and cholesterol were measured with colorimetric assays (TR22421 and TR13421, respectively, ThermoFisher Scientific, Middletown, VA, USA). Plasma ALT and AST concentrations were measured with kinetic spectrophotometric assays (A524-150 and A559-150, respectively, Teco Diagnostics, Anaheim, CA, USA).

\section{Liver TAG and glycogen assays}

Liver TAG concentrations were measured as performed previously [16]. 40-140mg of frozen tissue was homogenized using a bead homogenizer (Mini-Beadbeater, Biospec Products Inc., Bartlesville, OK, USA) in $0.9 \% \mathrm{NaCl}$ at a volume to provide $0.1 \mathrm{mg}$ liver/ $\mu \mathrm{L}$. Liver homogenate was combined (1:1) with $1 \%$ sodium deoxycholate, vortexed, and placed at $37^{\circ} \mathrm{C}$ for $5 \mathrm{~min}$ to solubilize 
lipids. TAG was then measured by colorimetric assay (TR22421, ThermoFisher Scientific, Middletown, VA, USA).

Liver glycogen was measured using previously described methods [29]. 20-75mg of liver tissue was boiled in $300 \mu \mathrm{L}$ of $30 \% \mathrm{KOH}$ at $100^{\circ} \mathrm{C}$ for $30 \mathrm{~min}$. Tubes were cooled on ice and $100 \mu \mathrm{L}$ of $2 \%$ $\mathrm{Na}_{2} \mathrm{SO}_{4}$ and $800 \mu \mathrm{L}$ of $100 \% \mathrm{EtOH}$ added and tubes vortexed. Tubes were boiled for $5 \mathrm{~min}$ and centrifuged at $16,000 \mathrm{x}$ g for $5 \mathrm{~min}$ then supernatant aspirated. The pellet was dissolved in $1 \mathrm{~mL}$ of $80 \%$ EtOH and recentrifuged $16,000 \mathrm{x} g$ for $5 \mathrm{~min}$. Final pellets were resuspended in $200 \mu \mathrm{L}$ of $0.3 \mathrm{mg} / \mathrm{mL}$ amyloglucosidase (Sigma, St. Louis, MO, USA) in 0.2M sodium acetate. Serial dilutions of $10 \mathrm{mg} / \mathrm{mL}$ oyster glycogen (Sigma, St. Louis, MO, USA) were prepared as standards. Samples and standards were incubated in a $40^{\circ} \mathrm{C}$ water bath for $3 \mathrm{~h}$, then diluted $1: 1$ with $\mathrm{H}_{2} \mathrm{O}$, and $5 \mu \mathrm{L}$ of each added to a 96-well plate. $200 \mu \mathrm{L}$ of glucose assay buffer (0.3 M Triethanolamine, $\mathrm{pH} 7.5,4 \mathrm{mM} \mathrm{MgCl} 2,2 \mathrm{mM} \mathrm{ATP}, 0.9 \mathrm{mM}$ $\mathrm{NADP}+$, and 5 $\mu \mathrm{g} / \mathrm{mL}$ Glucose-6-phosphate dehydrogenase) was added to each well, and absorbance measured at 340nm. $1 \mu \mathrm{g}$ of Hexokinase (Sigma, St. Louis, MO, USA) was then added to each well, the plate incubated at room temperature in the dark for $30 \mathrm{~min}$, and absorbance remeasured at 340nm.

\section{Pancreas and liver histology and immunohistochemistry analyses}

Formalin fixed liver and pancreas sections were embedded in paraffin blocks and sectioned onto glass slides. Liver slides were stained for H\&E and Sirius Red. Digitized liver slides were evaluated by a histopathologist blinded to mouse treatment and group designations, and scored for NAFLD activity (combined scores of steatosis, inflammation, and hepatocyte ballooning) and fibrosis similar to human biopsy scoring [30].

For the $d b / d b$ study, unstained pancreas slides were used for immunofluorescence as performed previously [27]. Slides were rehydrated then permeabilized with 1mg/mL trypsin (T7168, Sigma, St. Louis, $\mathrm{MO}$, USA) in $\mathrm{H}_{2} \mathrm{O}$ for $25 \mathrm{~min}$ at room temperature. Slides were washed $2 \times 5 \mathrm{~min}$ with $0.2 \%$ NP40-PBS, and blocked with $0.2 \%$ NP40-PBS containing 3\% BSA for 30min at room temperature. 
Slides were incubated with primary antibodies overnight at $4^{\circ} \mathrm{C}$ in a humidity chamber. Primary antibodies were guinea pig anti-insulin polyclonal and mouse anti-glucagon monoclonal, both 1:100 (ab7842 and ab10988, respectively, Abcam, Cambridge, MA, USA). The following day, slides were washed 3 x $5 \mathrm{~min}$ with $0.2 \%$ NP40-PBS, then incubated with secondary antibodies for $2 \mathrm{~h}$ at room temperature. Secondary antibodies were goat anti-guinea pig, Alexa Fluor488 and goat anti-mouse, Alexa Fluor594 (A11073 and A21125, respectively, ThermoFisher Scientific, Middletown, VA, USA). Slides were washed 3 x 5min with $0.2 \%$ NP40-PBS, and glass coverslips mounted with ProLong Diamond with DAPI (P36971, ThermoFisher Scientific, Middletown, VA, USA). Slides were imaged on a Leica DM5500B fluorescence microscope with Leica DFC365 FX camera (Leica Microsystems Inc., Buffalo Grove, IL, USA). Intensity of the green insulin fluorescence of pancreatic islets relative to nearby exocrine pancreas was measured using NIH Image J software.

For the MS-NASH study, pancreas slides were stained for H\&E or prepared for insulin immunohistochemistry by performing heat-induced epitope retrieval using EnVision FLEX Target Retrieval Solution, low pH 6 (Dako, K8005; Agilent, Santa Clara, CA, USA), with preheating to $80^{\circ} \mathrm{C}$ and increased temperature to $95^{\circ} \mathrm{C}$ for $20 \mathrm{~min}$ after slides were added. Slides were then incubated in $3 \%$ $\mathrm{H}_{2} \mathrm{O}_{2}$ for $5 \mathrm{~min}$ and incubated with rabbit anti-Insulin antibody, 1:100 for 45min (\#4590, Cell Signaling Technology, Danvers, MA, USA). Slides were then washed and secondary antibody was EnVision+ anti-rabbit labelled polymer-HRP applied for 30min (Dako, K4003; Agilent, Santa Clara, CA, USA), then $\mathrm{DAB}+$ chromogen solution for $5 \mathrm{~min}$. Slides were then rinsed in water and counterstained with modified Harris hematoxylin (Dako, S3301; Agilent, Santa Clara, CA, USA). Slides were cover-slipped and scanned at 20X on an Aperio Scanscope XT (Leica Biosystems, Buffalo Grove, IL, USA), and insulin+ cells quantified by HALO software (Indica Labs, Albuquerque, NM, USA).

\section{RNA isolation, cDNA synthesis, and quantitative RT-PCR analyses}

5-20mg of frozen liver tissue was homogenized in 1mL of RNA-STAT reagent (Tel-Test, Friendswood, TX, USA) with isopropanol and EtOH precipitation. RNA pellets were resuspended in 
200uL water and assessed by Nanodrop (ThermoFisher Scientific, Middletown, VA, USA). 1ug of RNA was reverse transcribed into cDNA by Superscript VILO kit (ThermoFisher Scientific, Middletown, VA, USA), using an Applied Biosystems 2720 Thermal Cycler (ThermoFisher Scientific, Middletown, VA, USA). qPCR was performed for all samples in duplicate with Power SYBR Green using an Applied Biosystems QuantStudio-3 real-time thermocycler (ThermoFisher Scientific, Middletown, VA, USA). Target gene $\mathrm{Ct}$ values were normalized to reference gene (RplpO) $\mathrm{Ct}$ values by the $2^{-\Delta \Delta \mathrm{Ct}}$ method. Oligonucleotide primer sequences are listed in electronic supplementary material Table 1.

\section{Statistics}

All data are expressed as means \pm SEM, with individual data points shown as dot plots, or curves over time displayed as means \pm SEM. Individual data points represent a single mouse. No data were excluded from any study measurements. Investigators were not blinded to genotype or treatment. The $d b / d b$ study was performed with 3 separate cohorts/replicates of mice and combined. For liver histology analysis of the MS-NASH studies, the pathologist was blinded to mouse number and treatment groups. The MSNASH study was performed with a single cohort of mice. Statistical analyses were performed using GraphPad Prism 8 (GraphPad, San Diego, CA, USA) using ordinary one-way ANOVA and Tukey's multiple comparisons tests and $p<0.05$ considered statistically significant.

\section{$\underline{\text { Results }}$}

\section{Effects of MSDC-0602K and Liraglutide on body weight, tissue weights, and glycemia of $d b / d b$} mice

Beginning at 9 weeks of age, $d b / d b$ mice were treated with either MSDC-0602K, Liraglutide, combination, or both vehicle solutions. 0602K-treatment caused slight weight gain and Lira-treatment caused slight weight loss compared to vehicle-treated $d b / d b$ mice (Fig. 1a). Surprisingly, mice treated with $0602 \mathrm{~K}+$ Lira combination displayed slight weight gain similar to mice treated with $0602 \mathrm{~K}$ alone (Fig. 1a). Blood glucose was monitored weekly and were completely corrected to $d b /+$ levels by $0602 \mathrm{~K}$ 
or $0602 \mathrm{~K}+$ Lira treatments, whereas Lira treatment more modestly improved glycemia (Fig. 1b). Both drugs individually improved glucose tolerance compared to vehicle-treated $d b / d b$ mice, and $0602 \mathrm{~K}+\mathrm{Lira}$ combination improved glucose tolerance beyond even lean $d b /+$ mouse levels (Fig. 1c,d). At sacrifice, 4h fasted blood glucose levels were completely corrected by 0602K or 0602K+Lira (Fig. 1e). Plasma fructosamine levels supported the weekly glucose measurements indicating that Lira modestly improved glycemia while $0602 \mathrm{~K}$ or $0602 \mathrm{~K}+$ Lira completely corrected glycemia (Fig. 1f). These results indicate that while $0602 \mathrm{~K}$ alone is able to correct glycemia, the combination of $0602 \mathrm{~K}+\mathrm{Lira}$ better improves glucose tolerance in $d b / d b$ mice.

Epididymal white adipose tissue (WAT) weights were unaffected by drug treatment (Fig. 2a), but $0602 \mathrm{~K}$ trended to increase inguinal subcutaneous WAT and significantly increased BAT weight whether provided alone or in combination with Lira (Fig. 2b,c). Increased liver weights in $d b / d b$ mice and not altered by drug treatment (Fig. 2d), yet hepatic TAG was nearly significantly reduced by 0602K+Lira (Fig. 2e). Hepatic glycogen levels were also increased in $d b / d b$ mice and were completely normalized by either $0602 \mathrm{~K}$ or $0602 \mathrm{~K}+\mathrm{Lira}$ (Fig. 2f). Thus, $0602 \mathrm{~K}$ increased adiposity and corrected hepatic glycogen levels whether given alone or in combination with Lira.

\section{Effects of MSDC-0602K and Liraglutide on insulinemia and plasma lipids}

Vehicle-treated $d b / d b$ mice were hyperinsulinemic and $0602 \mathrm{~K}$ decreased these plasma insulin concentrations (Fig. 3a). In contrast, Lira treatment further increased plasma insulin, yet mice treated with 0602K+Lira displayed reduced insulin similar to 0602K-treated animals (Fig. 3a). To resolve whether the changes in insulinemia were due to insulin secretion versus insulin clearance, we measured plasma C-peptide concentrations, which were strongly elevated in vehicle-treated $d b / d b$ mice. While Lira further increased C-peptide concentrations, $0602 \mathrm{~K}$ or $0602 \mathrm{~K}+$ Lira-treated mice displayed reduced C-peptide (Fig. 3b). An increased insulin/C-peptide ratio in 0602K-treated mice suggests that 0602K more strongly reduced insulin secretion versus increased clearance (Fig. 3c). Plasma NEFA, TAG, and cholesterol were all increased in vehicle-treated $d b / d b$ mice, and reduced by $0602 \mathrm{~K}$, Lira, or 
0602K+Lira treatments (Fig. 3d-f). These results suggest that 0602K reduces hyperinsulinemia by reducing insulin secretion even in combination with Liraglutide, yet all treatments were able to improve plasma lipids.

\section{MSDC-0602K improves islet insulin content by indirectly reducing insulin secretion}

Immunofluorescence of pancreas sections showed that the hyperinsulinemia of vehicle-treated $d b / d b$ mice caused a dramatic reduction of islet insulin content (Fig. 4a,b). 0602K-treatment alone or in combination with Lira significantly improved islet insulin content (Fig. 4a,b). We next wanted to test whether $0602 \mathrm{~K}$ was directly inhibiting insulin secretion. This is particularly intriguing as 0602K inhibits the MPC $[9,10]$, and we have previously shown that mice with beta cell-specific MPC-deletion display defective GSIS [27]. However, 0602K treatment of isolated islets had no effect on GSIS (Fig. 4c). Lastly, a single gavaged dose of $0602 \mathrm{~K}$ was able to reduce plasma insulin and C-peptide concentrations in diet-induced obese fl/fl (wildtype) and RIPCre-driven beta cell MPC2 knockout mice (Fig. 4d-f). Thus, insulin secretion was reduced by $0602 \mathrm{~K}$ even when the molecular target of $0602 \mathrm{~K}$ was not expressed in the pancreatic beta cells. Altogether, these results suggest that $0602 \mathrm{~K}$ preserves islet insulin content by indirectly reducing insulin secretion due to improved peripheral insulin sensitivity.

\section{MSDC-0602K and Liraglutide improve liver pathology in a mouse model of NASH}

Insulin resistance is a strong driver of NAFLD, and 0602K improves aspects of NASH histology in both mice and humans $[13,16]$. To test if NASH was more significantly improved with dual $0602 \mathrm{~K}$ and Lira therapy, MS-NASH mice were fed a western diet and provided fructose in the drinking water to develop obesity and NASH. Vehicle, 0602K, Lira, or 0602K+Lira treatments were started after 18 weeks on diet, and similar to the $d b / d b$ study, 0602K-treated mice tended to display increased body weight (Fig. 5a,b). Plasma ALT and AST were monitored monthly, and while Lira caused modest reductions in these plasma markers of liver injury, 0602K or 0602K+Lira induced more significant reductions (Fig. 5c-f). Only 0602K+Lira reduced liver weights compared to vehicle-treated mice, however as a percentage of body weight, 0602K- or 0602K+Lira-treated mice displayed reduced liver 
size (Fig. 6a,b). Liver TAG and glycogen concentrations were unaffected by any treatment in these mice

(Fig. 6c,d). Scoring of liver histology agreed with the biochemical measurements that steatosis was not improved by any treatment (Fig. 6e,f). However, hepatic inflammation and ballooning were significantly improved by combination 0602K+Lira treatment (Fig. 6g,h). Combined, while 0602K or Lira alone improved the NAFLD activity score, 0602K+Lira combination more significantly improved NASH liver histology (Fig. 6e,i). Thin bridging fibrosis was identified in all mice, and while the fibrosis histology scores were not improved by any treatment (data not shown), 0602K or 0602K+Lira treatment reduced the expression of several collagen and extracellular matrix-related genes (Fig. 6j). Altogether, these results indicate that combination of $0602 \mathrm{~K}$ and Lira improves NASH in this mouse model.

\section{MSDC-0602K decreases insulinemia and improves pancreas insulin content in MS-NASH mice}

0602K, Lira, or 0602K+Lira treatments did not reduce blood glucose in these MS-NASH mice

(Fig. 7a). However, 0602K or 0602K+Lira treatments strongly reduced plasma insulin (Fig. 7b). Plasma NEFA were also significantly reduced by $0602 \mathrm{~K}$ or $0602 \mathrm{~K}+$ Lira treatment (Fig. 7c), suggesting improved insulin action and decreased adipose lipolysis. Lastly, insulin immunohistochemistry identified a greater number of insulin+ cells in 0602K or 0602K+Lira treated pancreata (Fig. 7d,e). Thus, similar to the $d b / d b$ study, these results suggest that the improved insulin sensitivity with $0602 \mathrm{~K}$ increases islet insulin content.

\section{$\underline{\text { Discussion }}$}

The overall goal of these present studies was to assess whether combination of a novel TZD insulin sensitizer and a GLP-1RA would improve diabetes and NASH better than either individual therapy. Studies in $d b / d b$ mice suggested that combining MSDC-0602K and Liraglutide did indeed provide more significant improvement in glucose tolerance. Glycemia and insulinemia were greatly improved with $0602 \mathrm{~K}$ and not further improved by 0602K+Lira treatment. 0602K also improved pancreatic insulin content, and 0602K+Lira further increased islet insulin content. In MS-NASH mice, 
while plasma biomarkers and some aspects of NASH histology were improved by each treatment, more significant improvements were achieved with combination 0602K+Lira. A secondary goal of these studies was to test if the weight loss associated with Liraglutide could prevent the weight gain associated with TZDs. In the $d b / d b$ study, 0602K+Lira treated mice displayed similar slight weight gain as 0602Ktreated mice, however there was some attenuation of weight gain from combined 0602K+Lira treatment in the MS-NASH study. A study in diabetic rats using a rather low dose of Pioglitazone and double the dose of Lira as used in the current study also observed the greatest improvement in glucose tolerance with Pioglitazone+Lira, and while Lira-treated rats lost weight, Pioglitazone+Lira did not attenuate the Pioglitazone-induced weight gain [31]. However, a study of Pioglitazone+Lira in $d b / d b$ mice did observe attenuation of the Pioglitazone-induced weight gain with combined Lira treatment [32].

The strongest effects observed in our $d b / d b$ study were the complete correction of glycemia and reduction in insulinemia with $0602 \mathrm{~K}$. This decrease in insulinemia with TZDs has been well described in both humans and animal models of insulin resistance and diabetes [33-35]. The presiding dogma is that improved insulin sensitivity indirectly reduces the need for insulin hyper-secretion, and thus TZDs can improve beta cell insulin content and function [3, 35-37]. However, it has recently been recognized that traditional and PPAR $\gamma$-sparing TZDs can bind and inhibit the MPC [8-10], and pyruvate import into the mitochondria via the MPC plays an important role in beta cell glucose sensing and GSIS [27, 38]. This raises the possibility that MPC-inhibition with TZDs may directly inhibit beta cell insulin secretion, and indeed there are reports of acute TZD treatment decreasing GSIS [39, 40]. In this current study however, MSDC-0602K did not reduce GSIS in isolated islets (Fig. 4c), and 0602K was still able to reduce plasma insulin and C-peptide concentrations in mice lacking MPC expression in beta cells (Fig. 4e,f). A similar TZD molecule, MSDC-0160, was also found to have no effect on GSIS but improved insulin content and beta cell phenotype in isolated human islets [41]. Interestingly, MPC-inhibition with the tool-compound UK-5099 appears to alleviate the metabolic stress of islets cultured in high glucose [42]. Lastly, the preserved islet insulin content with TZDs can lead to enhanced insulin secretion during 
glucose challenge $[35,36]$. Altogether, these findings suggest that TZDs are not directly inhibiting GSIS, rather improved peripheral insulin sensitivity indirectly reduces the need for excessive insulin secretion.

While we observed similar decreases in insulinemia with $0602 \mathrm{~K}$ or $0602 \mathrm{~K}+\mathrm{Lira}$ in the MSNASH model of nonalcoholic steatohepatitis, the main goal of this experiment of course was to analyze fatty liver pathology. All treatments improved plasma ALT and AST, as well as certain aspects of liver histology, with the combination of $0602 \mathrm{~K}+$ Lira typically resulting in more significant improvements (Fig. 5c-f, Fig. 6e-i). Similar to our previous study in which we fed C57BL/6J mice a NASH-inducing diet [16], 0602K treatment did not improve hepatic triglycerides and steatosis (Fig. 6c,e,f). Conversely, steatosis was improved by $0602 \mathrm{~K}$ in humans with NASH [13]. Our previous study identified reductions in histologic fibrosis with 0602K [16], which were not observed in the current model, even with 0602K+Lira combination. Yet both the current and previous studies identified reduced expression of genes related to hepatic stellate cell activation and fibrosis, and these were not further reduced by 0602K+Lira (Fig. 6j).

There were a limited number of analyses that displayed additive improvement with combined 0602K+Lira treatment. Namely, glucose tolerance in the $d b / d b$ mice and liver weights and combined NAFLD activity scores in the MS-NASH mice were the main endpoints for which 0602K+Lira outperformed $0602 \mathrm{~K}$-only treatment. This could be due to a rather low dose of Liraglutide used in these studies $(0.2 \mathrm{mg} / \mathrm{kg}$ every-other-day), compared to other rodent studies that use similar doses with daily or even twice daily injections $[32,43,44]$. However, we are confident that we provided an effective dose of Liraglutide as Lira-treated animals displayed weight loss, increased plasma insulin concentrations, and improved glycemia. Most surprisingly, even though Lira increased insulin secretion, combination with $0602 \mathrm{~K}$ resulted in similar suppression of insulinemia compared to 0602K-treated mice with improved effects on glucose tolerance. 
In conclusion, while MSDC-0602K improved many aspects of insulin resistance and NASH by itself, glucose tolerance and several aspects of NASH were more significantly improved by combining 0602K and Liraglutide. In the MS-NASH study, Lira attenuated the weight gain associated with 0602K, but this was not the case in $d b / d b$ mice. This study also clarifies that TZDs likely reduce insulinemia not by directly inhibiting the MPC in beta cells but indirectly by improving peripheral insulin sensitivity. It remains unclear why MPC-inhibition with TZDs does not reduce GSIS unlike genetic MPC deletion [16]. Nonetheless, these studies suggest that combining insulin sensitizer and GLP-1RA therapies may better improve diabetes and fatty liver disease and potentially attenuate TZD-induced weight gain.

\section{$\underline{\text { Data availability }}$}

All data generated during these studies are included in the text, figures, and tables of this article and electronic supplementary material. Source data or materials will be supplied by the corresponding author with reasonable request.

\section{Funding}

K.S.M. is supported by NIH R00 HL136658. The $d b / d b$ study and analyses received no specific grant funding from any public or commercial agency. The MS-NASH mouse study was performed at Crown Biosciences Inc. and was funded by Cirius Therapeutics.

\section{Authors' relationships and activities}

J.R.C. is an employee, chief scientific officer, and stockholder of Cirius Therapeutics which is developing MSDC-0602K for NASH. All other authors declare no relationships or conflicts of interest.

\section{$\underline{\text { Contribution statement }}$}


bioRxiv preprint doi: https://doi.org/10.1101/2020.12.10.420190; this version posted December 14,2020 . The copyright holder for this preprint (which was not certified by peer review) is the author/funder, who has granted bioRxiv a license to display the preprint in perpetuity. It is made available under aCC-BY-ND 4.0 International license.

D.R.K., K.D.P., and M.S. performed the experiments, analyzed the data, assisted in manuscript writing and editing. L.H. analyzed the liver histology slides. J.R.C. conceived and designed the research, and wrote and edited the manuscript. K.S.M. conceived and designed the research, performed experiments, analyzed the data, and wrote and edited the manuscript. K.S.M. is the guarantor of this work and accepts full responsibility for the conduct of the performance and analyses of these studies, maintains full access to the data, and controlled the decision to publish.

\section{$\underline{\text { References }}$}

[1] Donath MY, Ehses JA, Maedler K, et al. (2005) Mechanisms of beta-cell death in type 2 diabetes. Diabetes 54 Suppl 2: S108-113.10.2337/diabetes.54.suppl_2.s108

[2] Talchai C, Xuan S, Lin HV, Sussel L, Accili D (2012) Pancreatic beta cell dedifferentiation as a mechanism of diabetic beta cell failure. Cell 150(6): 1223-1234. 10.1016/j.cell.2012.07.029

[3] Campbell IW, Mariz S (2007) Beta-cell preservation with thiazolidinediones. Diabetes Res Clin Pract 76(2): 163-176. 10.1016/j.diabres.2006.08.015

[4] Adams MD, Raman P, Judd RL (1998) Comparative effects of englitazone and glyburide on gluconeogenesis and glycolysis in the isolated perfused rat liver. Biochem Pharmacol 55(11): 19151920. S0006-2952(98)00052-5 [pii]

[5] Raman P, Foster SE, Stokes MC, Strenge JK, Judd RL (1998) Effect of troglitazone (Rezulin) on fructose 2,6-bisphosphate concentration and glucose metabolism in isolated rat hepatocytes. Life Sci 62(8): PL89-94

[6] Raman P, Judd RL (2000) Role of glucose and insulin in thiazolidinedione-induced alterations in hepatic gluconeogenesis. Eur J Pharmacol 409(1): 19-29

[7] Chen Z, Vigueira PA, Chambers KT, et al. (2012) Insulin resistance and metabolic derangements in obese mice are ameliorated by a novel peroxisome proliferator-activated receptor gamma-sparing thiazolidinedione. J Biol Chem 287(28): 23537-23548. 10.1074/jbc.M112.363960 [8] Colca JR, McDonald WG, Cavey GS, et al. (2013) Identification of a mitochondrial target of thiazolidinedione insulin sensitizers (mTOT)--relationship to newly identified mitochondrial pyruvate carrier proteins. PLoS One 8(5): e61551. 10.1371/journal.pone.0061551

[9] Divakaruni AS, Wiley SE, Rogers GW, et al. (2013) Thiazolidinediones are acute, specific inhibitors of the mitochondrial pyruvate carrier. Proc Natl Acad Sci U S A 110(14): 5422-5427.

10.1073/pnas. 1303360110

[10] McCommis KS, Chen Z, Fu X, et al. (2015) Loss of mitochondrial pyruvate carrier 2 in the liver leads to defects in gluconeogenesis and compensation via pyruvate-alanine cycling. Cell Metab 22(4): 682-694. 10.1016/j.cmet.2015.07.028

[11] Colca JR, VanderLugt JT, Adams WJ, et al. (2013) Clinical proof-of-concept study with MSDC0160, a prototype mTOT-modulating insulin sensitizer. Clin Pharmacol Ther 93(4): 352-359.

10.1038/clpt.2013.10

[12] Fukunaga T, Zou W, Rohatgi N, Colca JR, Teitelbaum SL (2015) An insulin-sensitizing thiazolidinedione, which minimally activates PPARgamma, does not cause bone loss. J Bone Miner Res 30(3): 481-488. 10.1002/jbmr.2364

[13] Harrison SA, Alkhouri N, Davison BA, et al. (2019) Insulin sensitizer MSDC-0602K in nonalcoholic steatohepatitis: A randomized, double-blind, placebo-controlled phase Ilb study. J Hepatol. 10.1016/j.jhep.2019.10.023 
[14] Vilsboll T, Zdravkovic M, Le-Thi T, et al. (2007) Liraglutide, a long-acting human glucagon-like peptide-1 analog, given as monotherapy significantly improves glycemic control and lowers body weight without risk of hypoglycemia in patients with type 2 diabetes. Diabetes Care 30(6): 1608-1610.

$10.2337 / \mathrm{dc} 06-2593$

[15] Secher A, Jelsing J, Baquero AF, et al. (2014) The arcuate nucleus mediates GLP-1 receptor agonist liraglutide-dependent weight loss. J Clin Invest 124(10): 4473-4488. 10.1172/JCI75276

[16] McCommis KS, Hodges WT, Brunt EM, et al. (2017) Targeting the mitochondrial pyruvate carrier attenuates fibrosis in a mouse model of nonalcoholic steatohepatitis. Hepatology 65(5): 15431556. 10.1002/hep.29025

[17] Cusi K, Orsak B, Bril F, et al. (2016) Long-Term Pioglitazone Treatment for Patients With Nonalcoholic Steatohepatitis and Prediabetes or Type 2 Diabetes Mellitus: A Randomized, Controlled Trial. Ann Intern Med. 10.7326/M15-1774

[18] Kalavalapalli S, Bril F, Koelmel JP, et al. (2018) Pioglitazone improves hepatic mitochondrial function in a mouse model of nonalcoholic steatohepatitis. Am J Physiol Endocrinol Metab.

10.1152/ajpendo.00023.2018

[19] Musso G, Cassader M, Paschetta E, Gambino R (2017) Thiazolidinediones and Advanced Liver Fibrosis in Nonalcoholic Steatohepatitis: A Meta-analysis. JAMA Intern Med.

10.1001/jamainternmed.2016.9607

[20] Neuschwander-Tetri BA, Brunt EM, Wehmeier KR, Oliver D, Bacon BR (2003) Improved nonalcoholic steatohepatitis after 48 weeks of treatment with the PPAR-gamma ligand rosiglitazone. Hepatology 38(4): 1008-1017. 10.1053/jhep.2003.50420

[21] Sanyal AJ, Chalasani N, Kowdley KV, et al. (2010) Pioglitazone, vitamin E, or placebo for nonalcoholic steatohepatitis. N Engl J Med 362(18): 1675-1685. 10.1056/NEJMoa0907929

[22] Somm E, Montandon SA, Loizides-Mangold U, et al. (2020) The GLP-1R agonist liraglutide limits hepatic lipotoxicity and inflammatory response in mice fed a methionine-choline deficient diet. Transl Res. 10.1016/j.trsl.2020.07.008

[23] Duparc T, Briand F, Trenteseaux C, et al. (2019) Liraglutide improves hepatic steatosis and metabolic dysfunctions in a 3-week dietary mouse model of nonalcoholic steatohepatitis. Am J Physiol Gastrointest Liver Physiol 317(4): G508-G517. 10.1152/ajpgi.00139.2019

[24] Eguchi Y, Kitajima Y, Hyogo H, et al. (2015) Pilot study of liraglutide effects in non-alcoholic steatohepatitis and non-alcoholic fatty liver disease with glucose intolerance in Japanese patients (LEAN-J). Hepatol Res 45(3): 269-278. 10.1111/hepr.12351

[25] Newsome PN, Buchholtz K, Cusi K, et al. (2020) A Placebo-Controlled Trial of Subcutaneous Semaglutide in Nonalcoholic Steatohepatitis. N Engl J Med. 10.1056/NEJMoa2028395

[26] (2011) Guide for the Care and Use of Laboratory Animals, 8th Edition. The National Academies Press (US). Washington (DC)

[27] McCommis KS, Hodges WT, Bricker DK, et al. (2016) An ancestral role for the mitochondrial pyruvate carrier in glucose-stimulated insulin secretion. Mol Metab 5(8): 602-614.

10.1016/j.molmet.2016.06.016

[28] Vigueira PA, McCommis KS, Schweitzer GG, et al. (2014) Mitochondrial pyruvate carrier 2 hypomorphism in mice leads to defects in glucose-stimulated insulin secretion. Cell Rep 7(6): 20422053. 10.1016/j.celrep.2014.05.017

[29] Suzuki Y, Lanner C, Kim JH, et al. (2001) Insulin control of glycogen metabolism in knockout mice lacking the muscle-specific protein phosphatase PP1G/RGL. Mol Cell Biol 21(8): 2683-2694. 10.1128/MCB.21.8.2683-2694.2001

[30] Brunt EM, Janney CG, Di Bisceglie AM, Neuschwander-Tetri BA, Bacon BR (1999) Nonalcoholic steatohepatitis: a proposal for grading and staging the histological lesions. Am J Gastroenterol 94(9): 2467-2474. 10.1111/j.1572-0241.1999.01377.x

[31] Larsen PJ, Wulff EM, Gotfredsen CF, et al. (2008) Combination of the insulin sensitizer, pioglitazone, and the long-acting GLP-1 human analog, liraglutide, exerts potent synergistic glucoselowering efficacy in severely diabetic ZDF rats. Diabetes Obes Metab 10(4): 301-311. 10.1111/j.14631326.2008.00865.x 
[32] Kimura T, Kaneto H, Shimoda M, et al. (2015) Protective effects of pioglitazone and/or liraglutide on pancreatic beta-cells in $\mathrm{db} / \mathrm{db}$ mice: Comparison of their effects between in an early and advanced stage of diabetes. Mol Cell Endocrinol 400: 78-89. 10.1016/j.mce.2014.11.018

[33] Miyazaki Y, Matsuda M, DeFronzo RA (2002) Dose-response effect of pioglitazone on insulin sensitivity and insulin secretion in type 2 diabetes. Diabetes Care 25(3): 517-523.

10.2337/diacare.25.3.517

[34] Tozzo E, Bhat G, Cheon K, Camacho RC (2015) Pioglitazone increases whole body insulin sensitivity in obese, insulin-resistant rhesus monkeys. PLoS One 10(5): e0126642.

10.1371/journal.pone.0126642

[35] Diani AR, Sawada G, Wyse B, Murray FT, Khan M (2004) Pioglitazone preserves pancreatic islet structure and insulin secretory function in three murine models of type 2 diabetes. Am J Physiol Endocrinol Metab 286(1): E116-122. 10.1152/ajpendo.00331.2003

[36] Qian X, Wang H, Yang G, et al. (2018) Pioglitazone Improved Insulin Sensitivity and First Phase Insulin Secretion Among Obese and Lean People with Diabetes: A Multicenter Clamp Study. Diabetes Ther 9(2): 815-826. 10.1007/s13300-018-0401-9

[37] Matsui J, Terauchi Y, Kubota N, et al. (2004) Pioglitazone reduces islet triglyceride content and restores impaired glucose-stimulated insulin secretion in heterozygous peroxisome proliferatoractivated receptor-gamma-deficient mice on a high-fat diet. Diabetes 53(11): 2844-2854. 10.2337/diabetes.53.11.2844

[38] Patterson JN, Cousteils K, Lou JW, Manning Fox JE, Macdonald PE, Joseph JW (2014) Mitochondrial Metabolism of Pyruvate Is Essential for Regulating Glucose-stimulated Insulin Secretion. J Biol Chem 289(19): 13335-13346. 10.1074/jbc.M113.521666

[39] Lamontagne J, Pepin E, Peyot ML, et al. (2009) Pioglitazone acutely reduces insulin secretion and causes metabolic deceleration of the pancreatic beta-cell at submaximal glucose concentrations. Endocrinology 150(8): 3465-3474. 10.1210/en.2008-1557

[40] Lamontagne J, Jalbert-Arsenault E, Pepin E, et al. (2013) Pioglitazone acutely reduces energy metabolism and insulin secretion in rats. Diabetes 62(6): 2122-2129. 10.2337/db12-0428

[41] Rohatgi N, Aly H, Marshall CA, et al. (2013) Novel insulin sensitizer modulates nutrient sensing pathways and maintains beta-cell phenotype in human islets. PLoS One 8(5): e62012.

10.1371/journal.pone.0062012

[42] Chareyron I, Christen S, Moco S, et al. (2020) Augmented mitochondrial energy metabolism is an early response to chronic glucose stress in human pancreatic beta cells. Diabetologia 63(12): 26282640. 10.1007/s00125-020-05275-5

[43] Buganova M, Pelantova $\mathrm{H}$, Holubova $\mathrm{M}$, et al. (2017) The effects of liraglutide in mice with dietinduced obesity studied by metabolomics. J Endocrinol 233(1): 93-104. 10.1530/JOE-16-0478

[44] Abdulreda MH, Rodriguez-Diaz R, Caicedo A, Berggren PO (2016) Liraglutide Compromises Pancreatic beta Cell Function in a Humanized Mouse Model. Cell Metab 23(3): 541-546.

10.1016/j.cmet.2016.01.009

\section{Figure legends}

Fig. 1. MSDC-0602K alone and in combination with Liraglutide improves glycemia and glucose tolerance in $d b / d b$ mice. (a) Average weekly body weights of each treatment group. (b) Average weekly blood glucose concentrations. (c,d) Blood glucose excursions and calculated area under the curve from an i.p. glucose tolerance test (GTT). (e) 4h fasted blood glucose concentrations measured at time of sacrifice. (f) Plasma fructosamine concentrations. $\mathrm{n}=7 \mathrm{db} /+\mathrm{Veh} ; 13 \mathrm{db} / \mathrm{db}$ Veh, $12 \mathrm{db} / \mathrm{db}$ 0602K, 7 
$d b / d b$ Lira, and $11 d b / d b 0602 \mathrm{~K}+$ Lira. All data are expressed as means \pm SEM or means \pm SEM within dot plot of individual data points. Individual data points represent a single mouse. Ordinary one-way ANOVA with Tukey's multiple comparison's test: $* p<0.05, * * p<0.01, * * * p<0.001$, and $* * * * p<0.0001$.

Fig. 2. Effects of MSDC-0602K or Liraglutide on tissue weights, liver triglycerides, and glycogen. (a-d) Epididymal (visceral) white adipose tissue (WAT), inguinal (subcutaneous) WAT, intrascapular brown adipose tissue (BAT) and liver weights. (e-f) Hepatic triglyceride (TAG) and glycogen concentrations. $\mathrm{n}=7 d b /+$ Veh; $13 d b / d b$ Veh, $12 d b / d b 0602 \mathrm{~K}, 7 d b / d b$ Lira, and $11 d b / d b 0602 \mathrm{~K}+$ Lira. All data are expressed measn \pm SEM within dot plot of individual data points. Individual data points represent a single mouse. Ordinary one-way ANOVA with Tukey's multiple comparison's test: * $p<0.05, * * p<0.01$, $* * * p<0.001$, and $* * * * p<0.0001$.

Fig. 3. MSDC-0602K reduces insulinemia and plasma lipids. (a-f) Plasma insulin, C-peptide, insulin/Cpeptide ratio, non-esterified fatty acids (NEFA), triglycerides (TAG), and cholesterol. $\mathrm{n}=7 \mathrm{db} /+\mathrm{Veh}$; $13 d b / d b$ Veh, $12 d b / d b$ 0602K, $7 d b / d b$ Lira, and $11 d b / d b$ 0602K+Lira. All data are expressed means \pm SEM within dot plot of individual data points. Individual data points represent a single mouse. Ordinary one-way ANOVA with Tukey's multiple comparison's test: *p<0.05, $* * p<0.01, * * * p<0.001$, and $* * * * p<0.0001$

Fig. 4. MSDC-0602K indirectly reduces insulin secretion and improves islet insulin content. (a) Representative H\&E images and insulin/glucagon immunofluorescence of pancreatic islets. (b) Islet insulin fluorescence intensity measured by the islet green fluorescence normalized to green fluorescence of surrounding exocrine pancreas tissue. Individual data points represent the average intensity of at least 12 islets per mouse. (c) Insulin secretion from isolated wildtype islets at 1 versus $23 \mathrm{mM}$ glucose comparing DMSO vehicle with $10 \mu \mathrm{M}$ MSDC-0602K treatment. Individual data points represent the 
average of an independent experiment, each containing 3-4 individual replicates. (d) Average weekly body weights of fl/fl (wildtype) and beta cell specific MPC2 knockout (RIPCreMPC2-/-) mice fed highfat diet for 10 weeks. After 10 weeks of high-fat diet, mice were gavaged once with vehicle or $30 \mathrm{mg} / \mathrm{kg}$ MSDC-0602K and euthanized the following morning after $~ 16$ hours. (e,f) Plasma insulin and C-peptide measured after a single dose of vehicle or $0602 \mathrm{~K}$. All data are expressed as means \pm SEM or means \pm SEM within dot plot of individual data points. Individual data points represent a single mouse. Ordinary oneway ANOVA with Tukey's multiple comparison's test: ${ }^{*} p<0.05, * * p<0.01, * * * p<0.001$, and $* * * * p<0.0001$

Fig. 5. MSDC-0602K and Liraglutide improve plasma measures of liver injury. (a,b) Average weekly body weight (BW) and final BW of MS-NASH mice treated with either Vehicle, 0602K, Liraglutide, or 0602K+Lira. (c-f) Average monthly plasma alanine aminotransferase (ALT) and aspartate aminotransferade (AST) and final ALT and AST. n=10 for all groups. All data curves are expressed as means \pm SEM, and final data expressed as means \pm SEM within dot plot of individual data points. Individual data points represent a single mouse. Ordinary one-way ANOVA with Tukey's multiple comparison's test: $* p<0.05, * * p<0.01, * * * p<0.001$, and $* * * * p<0.0001$.

Fig. 6. MSDC-0602K+Liraglutide treatment improves NASH liver pathology. (a,b) Liver weight and liver weight normalized to body weight (BW). (c,d) Liver triglyceride (TAG) and glycogen concentrations. (e) Representative liver H\&E and Sirius Red histology images. (f-i) Liver histology scoring for NAFLD (steatosis, inflammation, hepatocyte ballooning, and combined NAFLD activity score) determined by a histopathologist blinded to treatment groups. (j) Hepatic gene expression for genes related to hepatic stellate cell activation, collagen, and extracellular matrix organization. $n=10$ for all groups. All data expressed as means \pm SEM within dot plot of individual data points. Individual data 
points represent a single mouse. Ordinary one-way ANOVA with Tukey's multiple comparison's test:

${ }^{*} p<0.05, * * p<0.01, * * * p<0.001$, and $* * * * p<0.0001$

Fig. 7. MSDC-0602K improves insulinemia and islet pancreatic insulin content in MS-NASH mice. (a-

c) Blood glucose concentrations, plasma insulin concentrations, and plasma non-esterified fatty acids

(NEFA). (d,e) Percentage of insulin positive cells in pancreas sections and representative pancreas H\&E histology and insulin immunohistochemistry images. $\mathrm{n}=10$ for all groups. All data expressed as means \pm SEM within dot plot of individual data points. Individual data points represent a single mouse.

Ordinary one-way ANOVA with Tukey's multiple comparison's test: ${ }^{*} p<0.05,{ }^{* *} p<0.01,{ }^{*} * *^{*}<0.001$, and $* * * * p<0.0001$ 


\section{Figure 2}
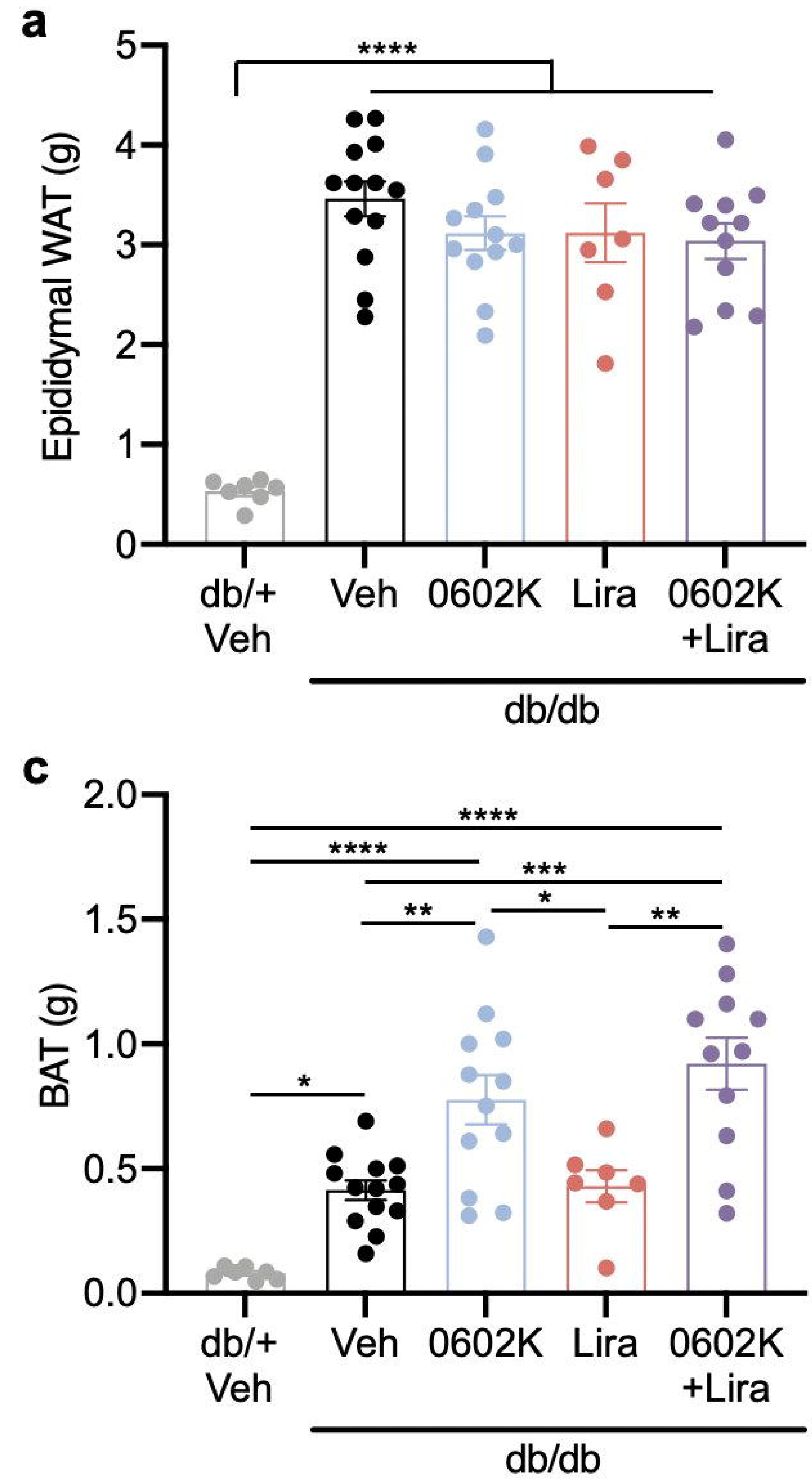

e

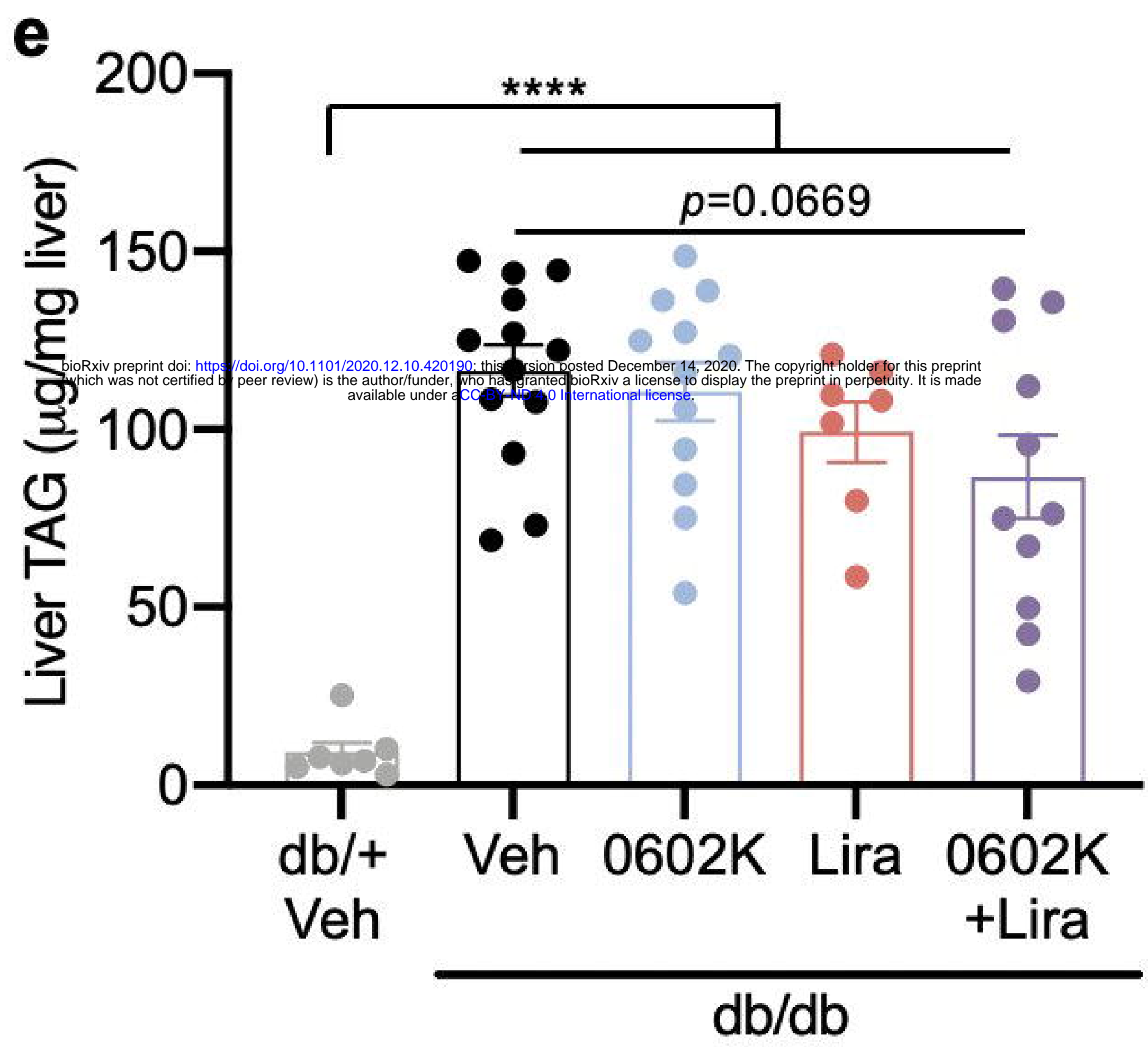

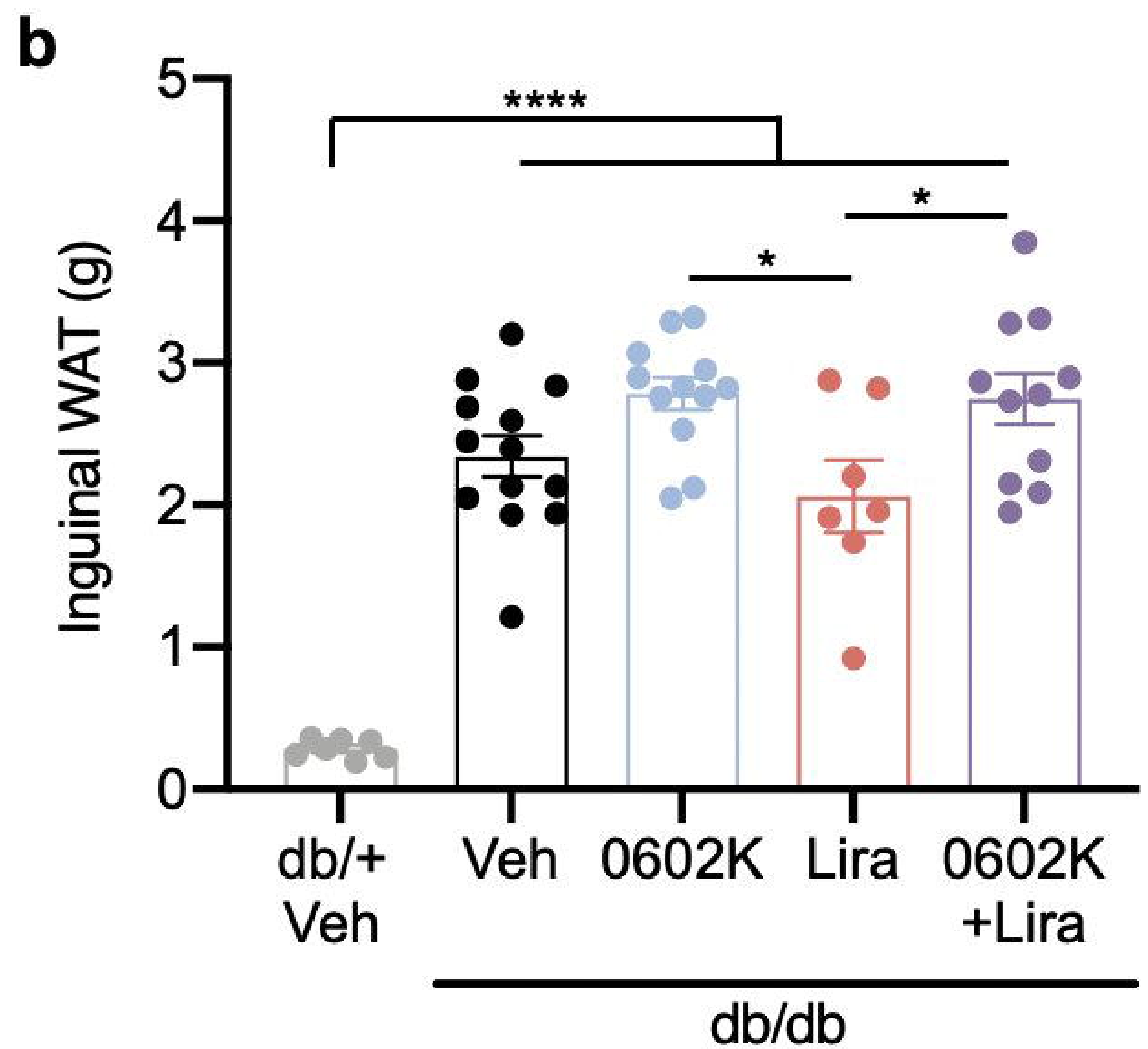
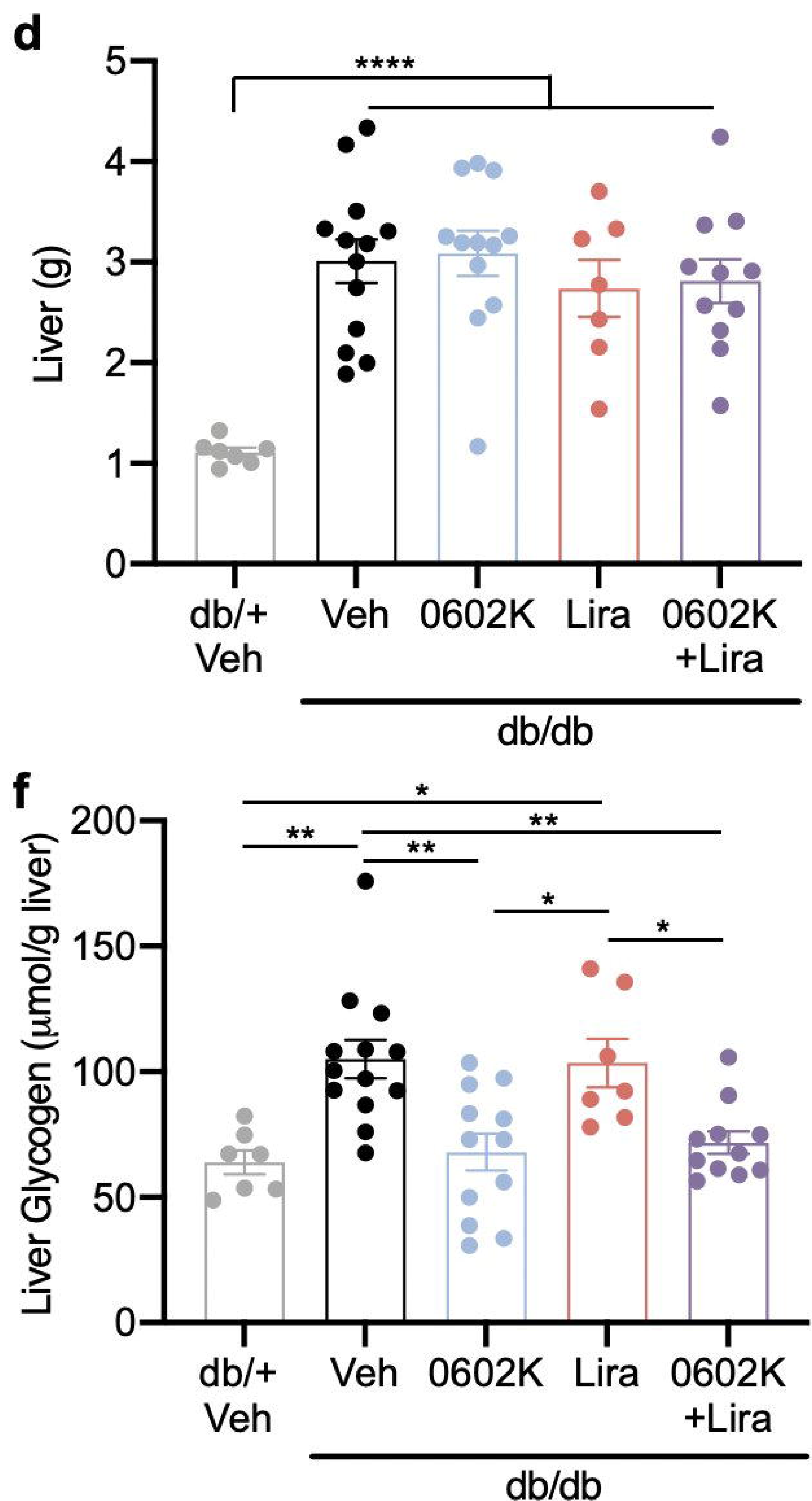

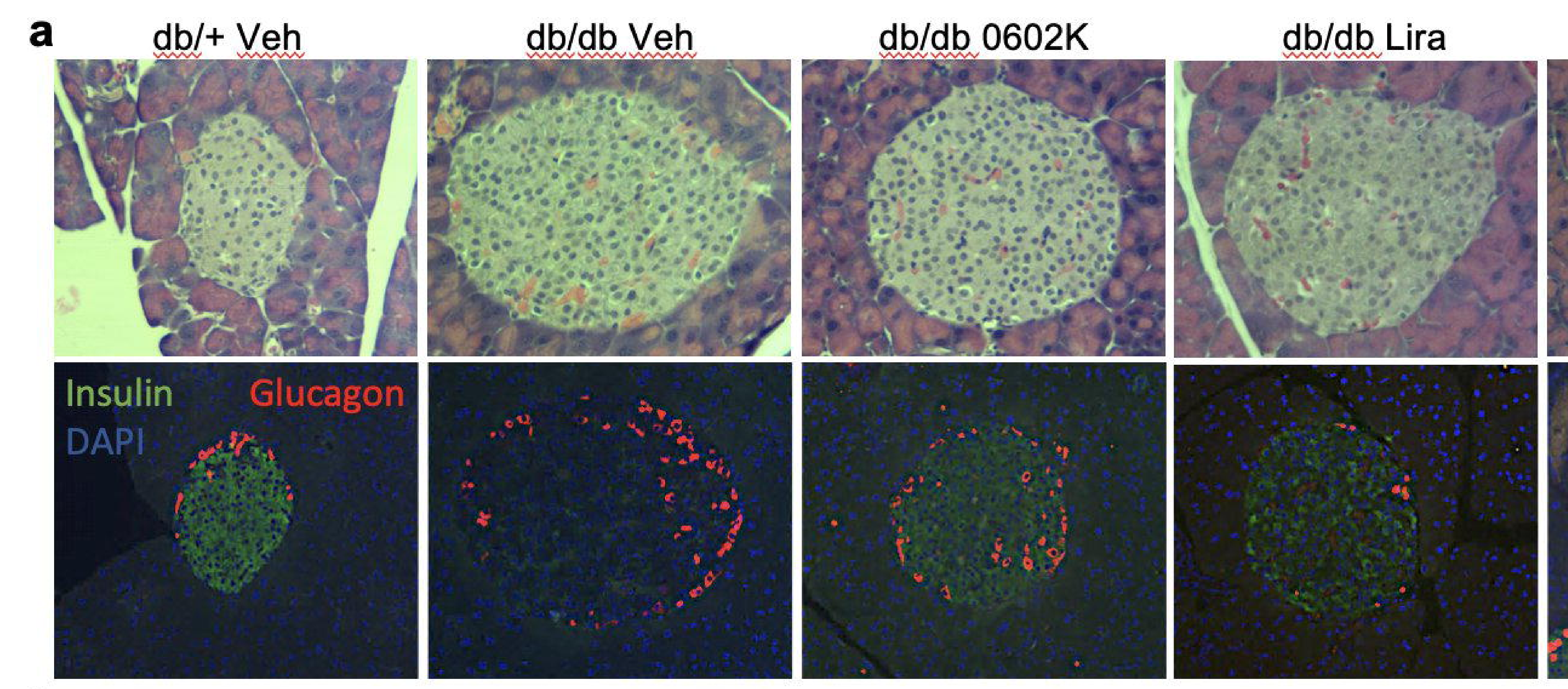

$\mathrm{db} / \mathrm{db}$ 0602K+Lira

b

b
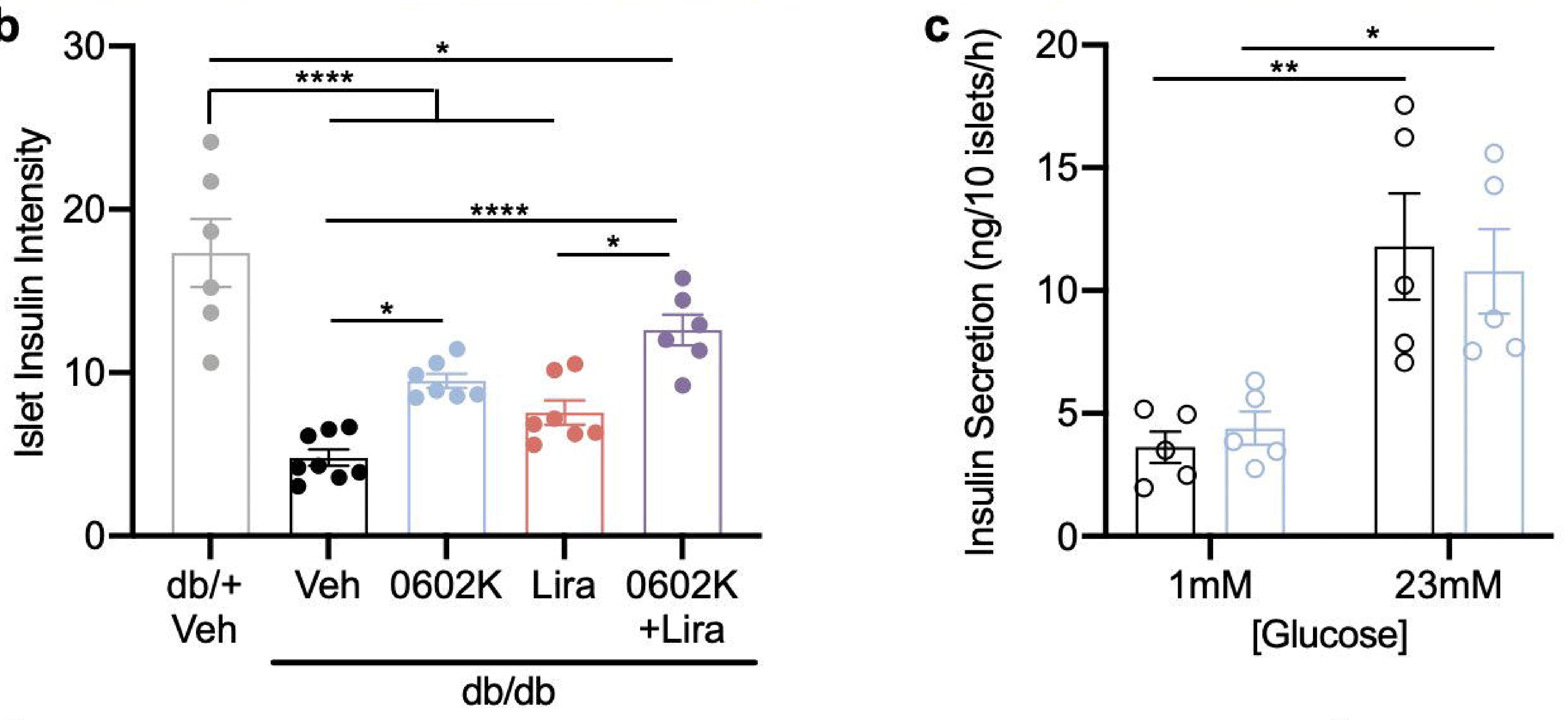

[Glucose]

- Veh

- $10 \mu \mathrm{M} 0602 \mathrm{~K}$
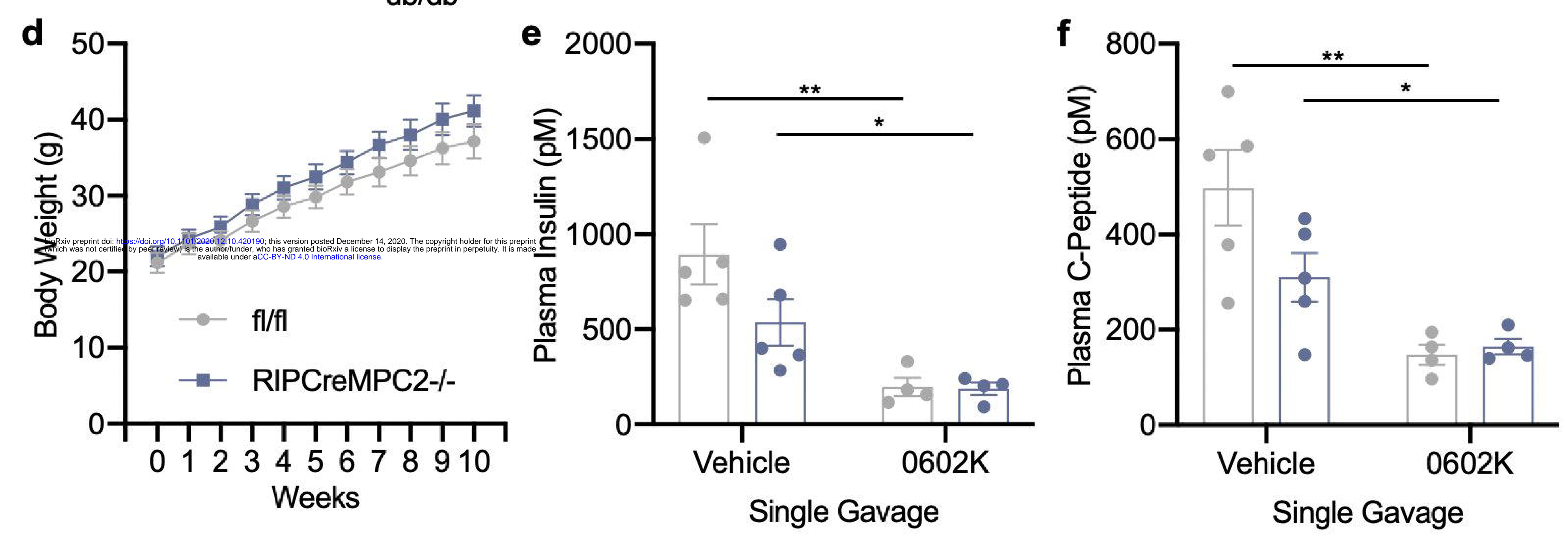

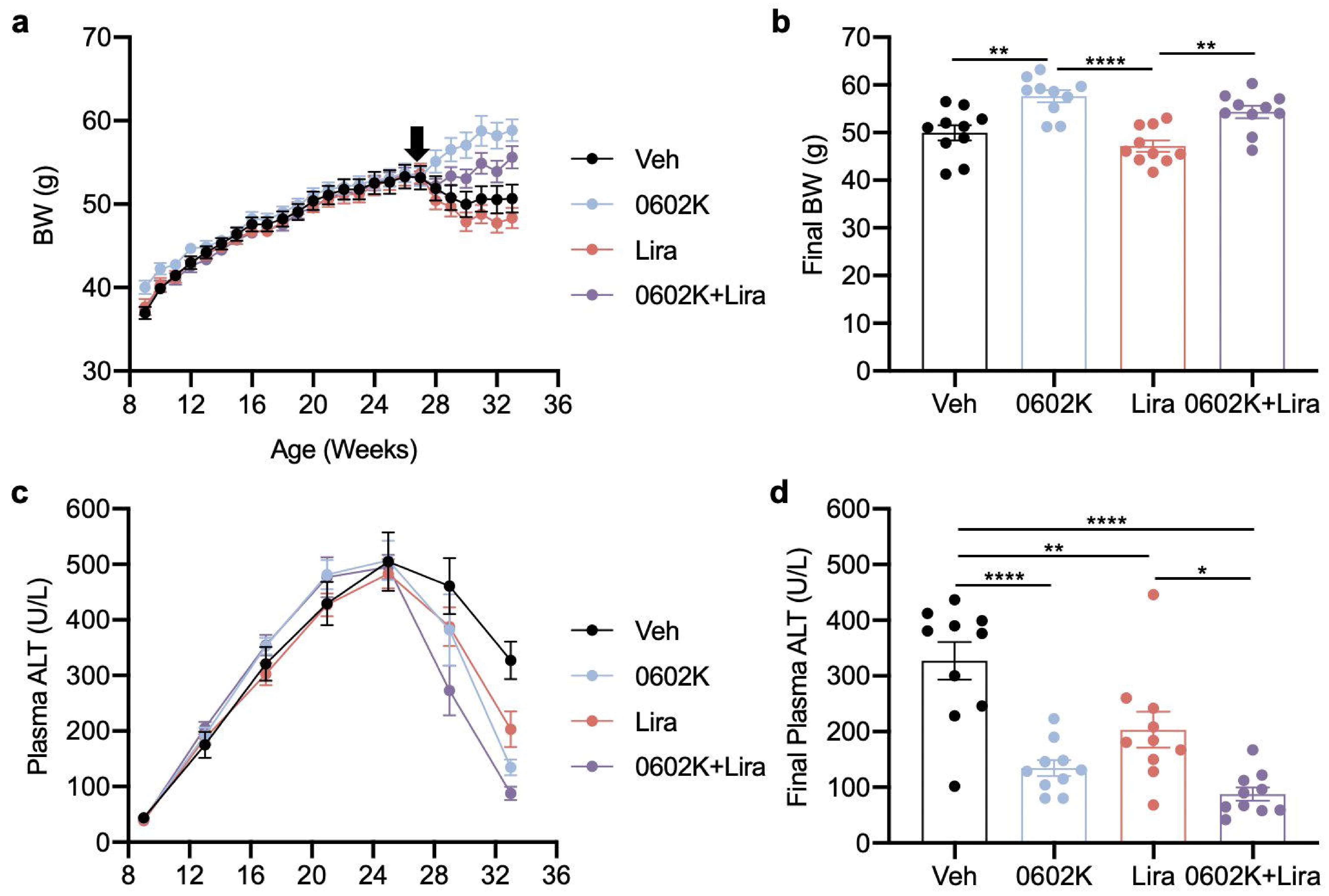

Age (Weeks)
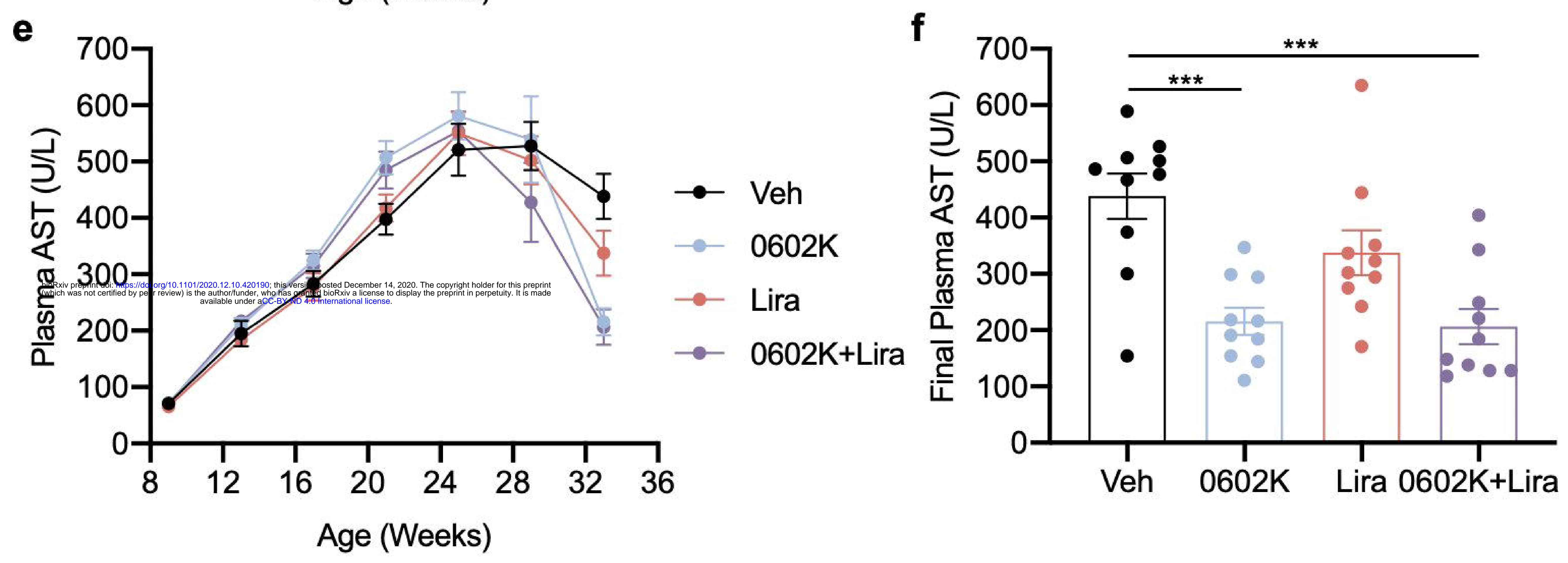
Figure 6
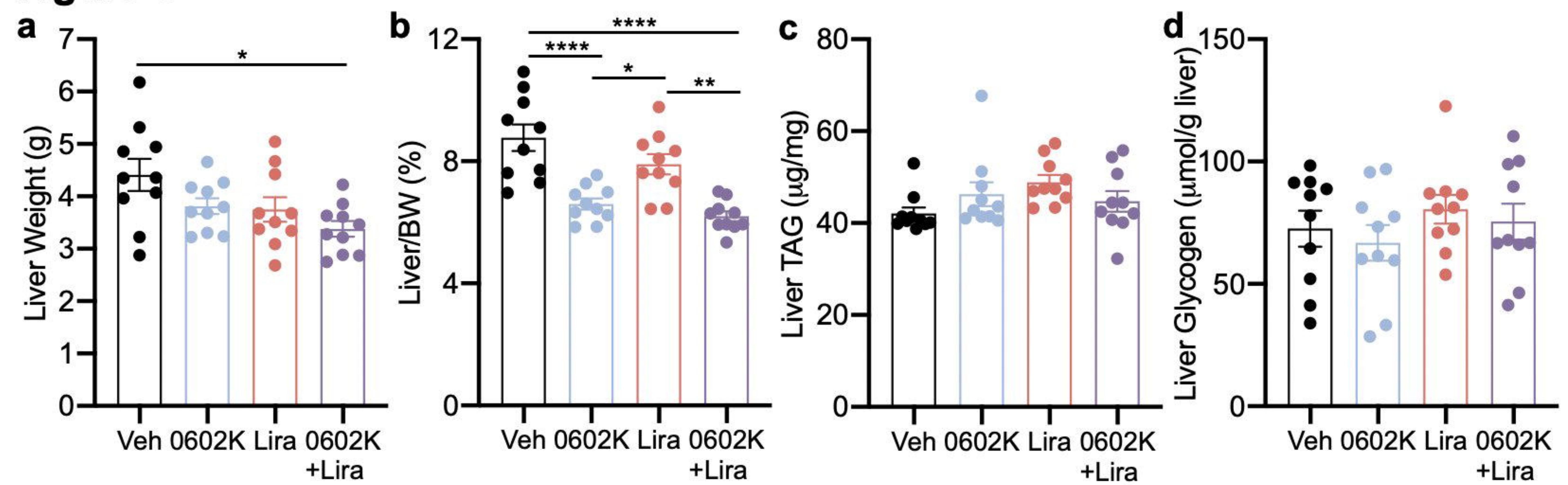

e

Vehicle

0602K

Lira

0602K+Lira
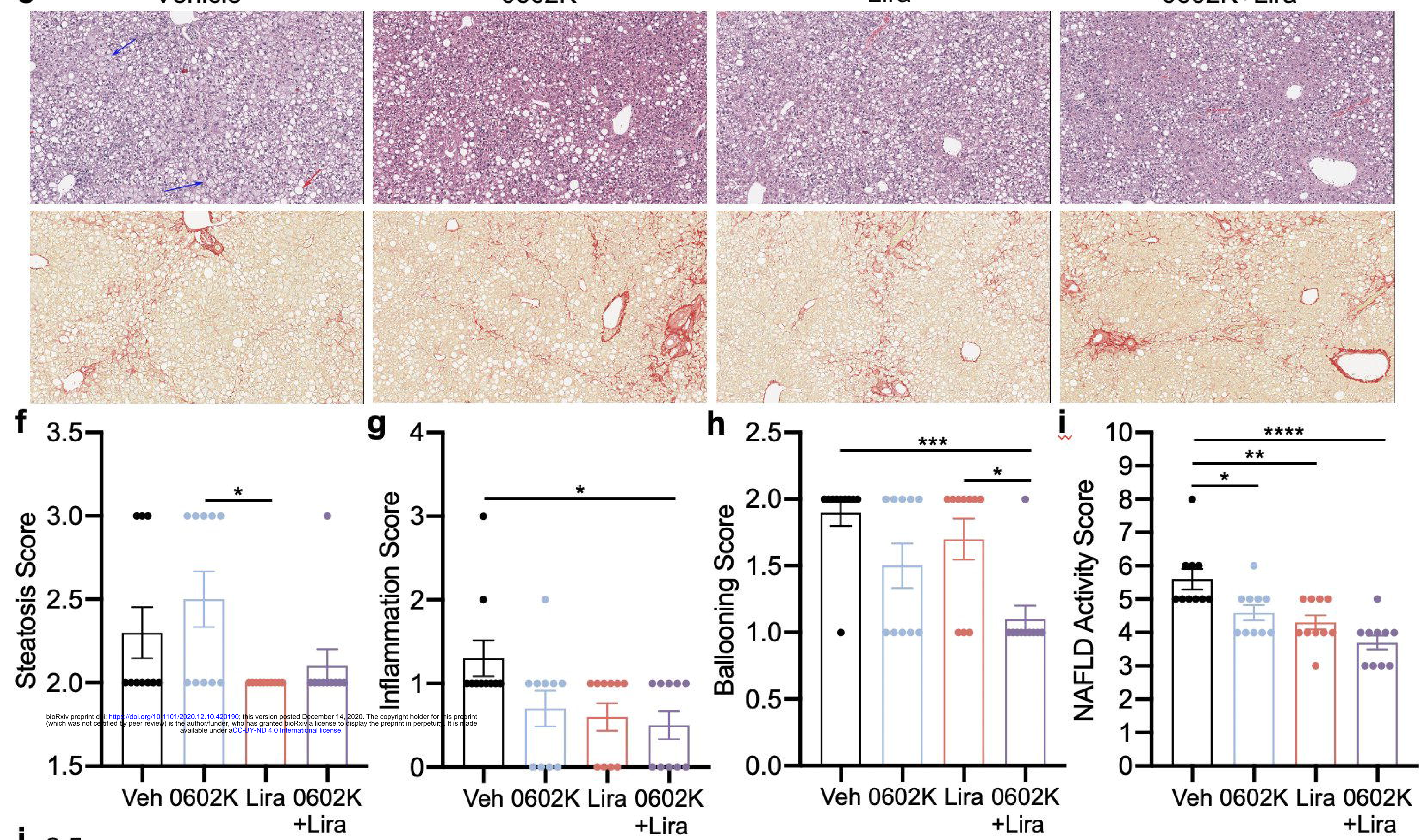

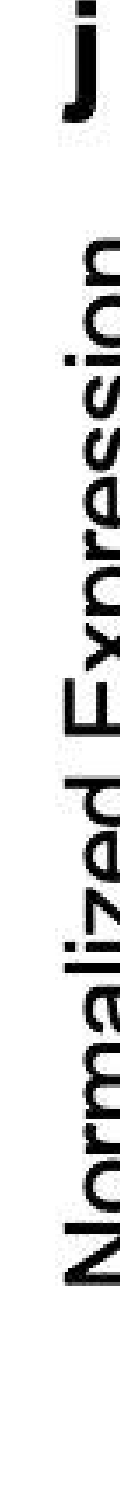
1.0 0.0
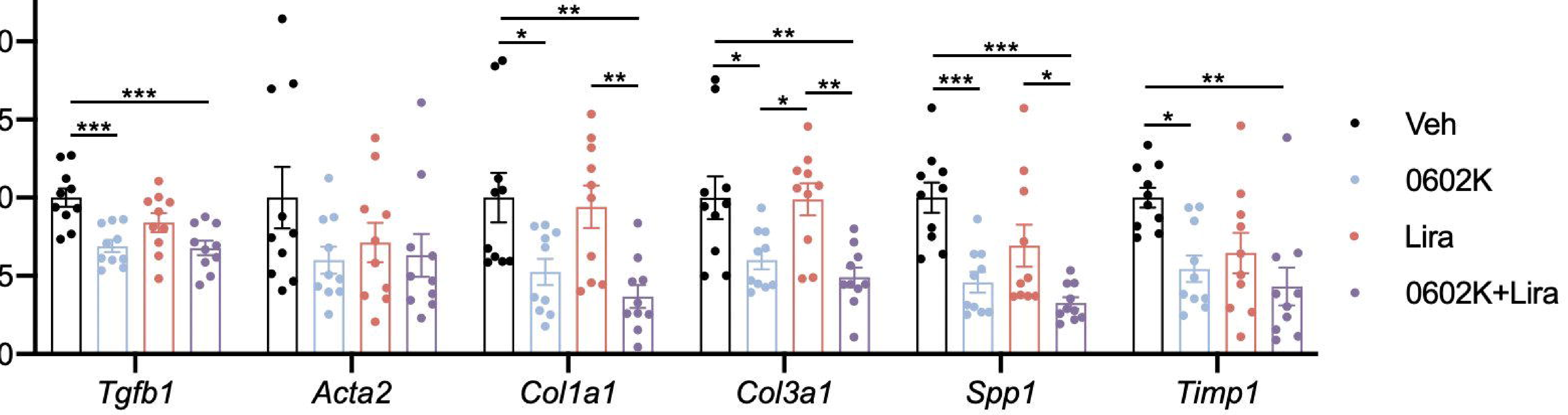
